\title{
Laparoscopic treatment of lymphoceles after renal transplantation
}

Marcelo Lopes de Lima, Cristiano Augusto Calderaro Cotrim, Juliano Cesar Moro, Ricardo Miyaoka, Carlos Arturo Levi D’Ancona

Division of Urology, Department of Surgery, State University of Campinas (UNICAMP), Campinas, Sao Paulo, Brazil

\section{ABSTRACT}

Objective: Lymphocele formation following renal transplantation is a frequent complication and may affect as many as $49 \%$ of patients. Operative treatment of symptomatic post transplant lymphocele (PTL) consists of wide drainage of the fluid collection into the abdominal cavity by excising its wall, connecting the lymphocele cavity to the intraperitoneal space. Laparoscopic fenestration seems to be the best treatment as it combines satisfying success rates with a minimally invasive approach. The aim of the study was to review a single center experience on the laparoscopic treatment of symptomatic PTL and detail relevant aspects of the surgical technique.

Materials and Methods: The data of 25 patients who underwent laparoscopic surgical treatment for a symptomatic lymphocele following kidney transplantation were retrospectively reviewed. Demographic data and surgical results were assessed. Detailed surgical technique is provided.

Results: Between 1996 and 2008, 991 patients received a kidney transplant at our institution. Twenty-five patients (2.52\%) developed a symptomatic lymphocele and laparoscopic drainage was performed. The indications for surgical drainage were graft dysfunction (84\%), local symptoms (16\%) or both (32\%). The mean time until surgical therapy was $14.2 \pm 6$ weeks. Mean hospital stay was $1.5 \pm 0.2$ days. Postoperative complications occurred in only 2 patients (8\%) (one ureteral injury and one incisional hernia) and required reoperation. After a mean followup of $36.2 \pm 4$ months, only 1 patient had a symptomatic recurrence.

Conclusions: Laparoscopic fenestration is an effective surgical technique to treat symptomatic lymphocele following kidney transplantation with low recurrence rate and long standing results.

\section{ARTICLE INFO}

\section{Key words:}

Kidney transplantation; lymphocele; laparoscopy; Surgical Procedures;

Minimally Invasive

Int Braz J Urol. 2012; 38: 215-21

Submitted for publication: July 20, 2011

Accepted after revision: October 10, 2011

\section{INTRODUCTION}

Lymph is the result of blood plasma extravasation from the capillary vessels into the interstitial space. Its role is related to the maintenance of adequate human body organs and tissues perfusion and depends on the balance between hydrostatic and coloidosmotic pressures both inside and outside the capillary vessels (1).

Lymphocele is a pseudocystic entity with lymph content covered with a hard fibrous capsule. It can be a complication of any surgery involving the lymphatic system. The term "lymphocele" (lymphatic collection without an epithelial 
lining) originated from the Japanese literature. Medical and surgical factors are involved in the etiology of the lymphocele. The surgical factor appears to be the most important, specially the lesion of the allograft's lymphatic vessels, and also of the perivascular ones near the anastomosis $(2,3)$.

Liquid collections around the renal allograft are very frequent and may occur in up to $49 \%$ of patients. The significant majority of post transplant lymphoceles occurs within the first 3 months after surgery (4). Although most collections are asymptomatic and resolve spontaneously, some may lead to more serious complications. Some of these patients may have general, vascular or obstructive uropathy repercussions that are secondary to the collection, and require early treatment to preserve allograft function (5). These repercussions cause symptoms that result from compression of the pressure created by the lymphocele on the ureter, bladder, or vascular structures, or from infection of these collections. Intervention is indicated when the compressive effect causes graft dysfunction or other symptoms including deep vein thrombosis, abdominal pain, or edema of the lower extremity and genitalia (6).

In this scenario, laparoscopy allows a minimally invasive approach combining image magnification, precise lymphocele drainage and minimal morbidity when proper surgical technique is implemented.

We reviewed our single center 12 - year experience with symptomatic post transplant lymphocele to evaluate the efficacy and safety of laparoscopic procedure for this problem. A comparison with other centers results is provided.

\section{MATERIALS AND METHODS}

After approval by the Institutional Review Board, 991 adult patients who underwent kidney transplantation at our institution between March 1996 and July 2008 were evaluated. The information regarding patients and associated surgical procedures were recorded.

In this study, only patients who presented either allograft dysfunction or local symptoms with perirenal and perivesical fluid collection surrounding the kidneys or ureter were considered. They were observed through ultrasonographic evaluation in order to confirm the presence of lymph and the absence of urine. A sample of the fluid collection was acquired through ultrasound guided percutaneous puncture and sent for biochemical analysis. Creatinine level similar to serum confirmed its lymphatic nature. Computer tomography (CT scan) provided better evaluation of lymphocele collection and its anatomical relations to the surrounding structures.

All drainage procedures were performed under general anesthesia and a single dose of antibiotics (first generation cephalosporin) was given for prophylaxis.

A transperitoneal three-port technique was used. Pneumoperitoneum was obtained with $\mathrm{CO}_{2}$ insuflation up to a pressure of $12 \mathrm{mmHg}$ following the insertion of a Verress needle through the supraumbilical crease. One $10-\mathrm{mm}$ camera port was placed in the supraumbilical location followed by a second 5-mm working port along the mid clavicular line near the costal margin on the transplant ipsilateral side and a third 5-mm port in the opposite flank side.

The essential steps of the procedure include: identification of the limits of the lymphocele, laparoscopic needle aspiration for confirmation; and finally precise incision of the lymphocele cavity. The common wall between the lymphocele and the peritoneal cavity was excised with electrocautery after determining the location of the kidney and other vital structures. The fenestration created between the extraperitoneal space containing the transplanted kidney associated lymphocele and the peritoneal cavity was made as large as possible but with great care to avoid the inferior and lateral peritoneal surfaces where the ureter and renal hylum could be accidentally injured. In this scenario, critical care was taken when performing the lymphocele wall incision (Figure-1). The cutting line was always longitudinal in a cranial to caudal fashion adding extra care to prevent ureteral and vascular injuries.

Patients were encouraged to ambulate and were started on a diet on the first day after surgery. 
Figure 1 - Transperitoneal three-port position and lymphocele wall incision.

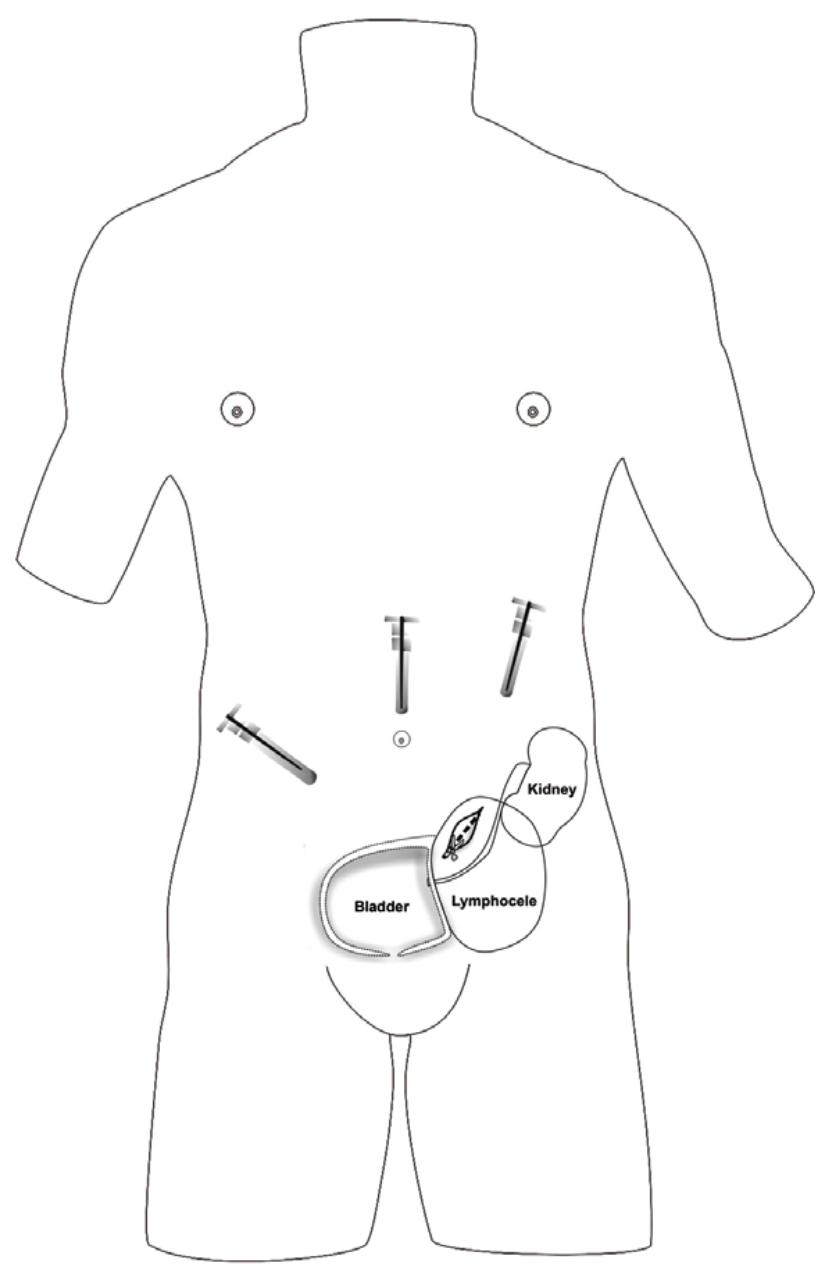

\section{RESULTS}

During the study period, 991 adult renal transplants (523 cadaveric, 468 living) were performed (Table-1). The incidence of symptomatic lymphoceles was $2.52 \%$. There were 25 lymphoceles treated (14 women and 11 men). Preoperative confirmation of the nature of the fluid collection was obtained in all cases by percutaneous aspiration and fluid analysis including creatinine and cytology with differential cells and bacterial culture. There was no evidence of infection in any case of our cohort. The indications for surgical drainage were graft dysfunction (21 pts. - 84\%), local symptoms (4 pts. $-16 \%$ ) or both (8 pts. - 32\%) (Table-1). The mean time from transplantation to surgical therapy for lymphocele was $14.2 \pm 6$ weeks.

The operative time (including anesthesia time) was $88 \pm 6 \mathrm{~min}$. There were no intraoperative complications and all cases could be completed with pure laparoscopic technique. Mean hospital stay was $1.5 \pm 0.8$ days (Table- 2 ).

Clinical follow-up included serum creatinine measurements and abdominal ultrasound after definitive surgical treatment. One patient (4\%) developed symptomatic recurrence after 45 days following lymphocele treatment and underwent reoperation. An open technique approach was chosen in this case as technical difficulty was anticipated since the patient had had several abdominal surgical interventions other than the kidney transplantation and lymphocele drainage. Preoperative imaging allowed for location near to the graft hylum. Confirmation of proper site was carried out only with palpation and careful aspiration. This patient underwent uncomplicated repeated fenestration without subsequent recurrence. Two patients $(8 \%)$ had postoperative complications (one ureteral injury and one incisional hernia) that required reoperation. The patient with ureteral injury developed symptomatic abdominal fluid collection one week after the procedure. In the reoperation, a lateral opening at mid third of transplanted ureter $1 \mathrm{~cm}$ away from the drainage incision could be noted. Correction was undertaken with ureteral edges revitalization and single stitches with absorbable suture. A double $\mathrm{J}$ catheter was placed under fluoroscopic control as well.

Incisional hernia developed 2 weeks after laparoscopy. An intestinal segment could be noted through the hernia ostium at the time of reoperation. All patients restored renal function after definitive treatment.

\section{DISCUSSION}

Perinephric fluid collections are common in the early postoperative period. Whereas asymptomatic fluid collections do not require aggressive diagnostic workup or treatment, symptomatic lymphoceles require definitive treatment (7). 
Table 1 - Patient demographics.

\begin{tabular}{lc}
\hline Number of transplants (living/cadaveric donors) & $991(468 / 523)$ \\
\hline Number of post transplant lymphoceles & $25(2.52 \%)$ \\
Number of lymphoceles (living/cadaveric donors) & $8 / 17$ \\
Mean interval from transplantation (weeks) & $14.2 \pm 6$ \\
Mean diameter of lymphoceles (centimeters) & $15.4 \pm 2.8$ \\
Number women/men & $14 / 11$ \\
Mean age (years) & $38 \pm 9$ \\
Operative time (minutes) & $88 \pm 6$ \\
Intraoperative complications & $\mathrm{No}$ \\
Conversion & $\mathrm{No}$ \\
Hospital stay (days) & $1.5 \pm 0.2$ \\
Follow up (months) & 36.2 \\
Number of symptomatic recurrences & $1(4 \%)$ \\
Postoperative complications & $2(8 \%)$ \\
\hline
\end{tabular}

Table 2 - Intraoperative data and outcome.

\begin{tabular}{lc}
\hline Operative time & $88 \pm 6$ min \\
\hline Intraoperative complications & No \\
Conversion & No \\
Hospital stay (days) & $1.5 \pm 0.2$ \\
Follow up (months) & 36.2 \\
Number of symptomatic recurrences & $1(4 \%)$ \\
Postoperative complications & $2(8 \%)$ \\
\hline
\end{tabular}

Symptomatic lymphoceles are of much less frequency but are easily recognized and diagnosed (8). When symptoms occur they are typically related to compression of adjacent structures. The most frequent symptoms associated with lymphoceles are lower abdominal pain, lower extremity or scrotal swelling, deep vein thrombosis, or graft dysfunction secondary to ureteral or bladder outlet obstruction (1). In
$84 \%$ of the patients of our series increased serum creatinine level was the first symptom indicative of a lymphocele. Lower extremity edema, genital edema, deep venous thrombosis before detection of the lymphocele were the leading symptoms in only $16 \%$ of the patients.

In our series of 991 patients studied over 12 years, the incidence of lymphocele was $2.52 \%$, which is comparable to the rate of $1 \%$ to $12 \%$ of patients observed in other reviews (8). The occurrence of lymphoceles after surgery for kidney transplantation does not seem to be the result of any single factor (9). A number of aetiological factors are described and a large variety of combinations are likely to be causal (9). Like illustrated previously there are good evidences to suggest that the extraperitoneal location of renal transplants and subsequent lack of communication with the absorptive peritoneal surface contribute to significant elevated incidence of lymphocele after renal transplantation. Other factors can contribute to lymphocele formation like delayed graft function (10), repeat transplantation (11), acute graft rejection (12), cadaveric 
donor kidney (3), sirolimus immunosuppression (13), and other host disease factors such as adult polycystic kidney disease (14).

Thromboembolic prophylaxis with lowdose subcutaneous heparin can be another aetiological factor. This may be a consequence of the higher vulnerability of the lymphatic system to the effects of anticoagulants due to the lower concentration of clotting factors and the lack of platelets (9).

Although it has been suggested that implantation to the external iliacs is a risk factor for lymphocele formation, we routinely use the internal or external iliacs and did not find this to be a problem (15).

In this series, there was a significant difference in the number of lymphoceles in the kidneys from cadaveric donors. It could be explained by bigger inflammatory process in these patients, where surgery performed in urgency and not routinely like living donors.

Renal transplant recipients with increased serum creatinine levels and clinical symptoms indicative of a lymphocele should undergo diagnostic ultrasound or computerized tomography.

Before the laparoscopic era the only alternative to open transperitoneal lymphocele drainage was percutaneous aspiration with subsequent observation or sclerosant therapy. They introduce the possibility of infection and they can keep open lymphatic vessels due to continuous aspiration (16).

Closed percutaneous drainage systems can be used for a defined period of time. They also carry a recurrence rate of 40-50\%. A number of sclerosants have been used as an adjunct to percutaneous drainage (3). Although sclerosant therapy has a better success rate than simple aspiration, recurrences remain common, and complications including acute renal failure have been reported (17). No large series have established the benefit of one method of sclerosis over the others (4).

Operative treatment of symptomatic post transplant lymphocele consists of lymphocelectomy with creation of a large internal window for intraperitoneal fluid drainage. Open lymphocelectomy has long been the standard. A literature review including 129 patients who underwent open drainage between 1980 and 1998 revealed a complication rate of $4 \%$ and a recurrence rate of $15 \%$ (18). It is believed that the high incidence of symptomatic recurrences following open drainage is a result of bowel adhesions obstructing the internal window and thus causing extraperitoneal fluid accumulation.

Since laparoscopic fenestration of post transplant lymphocele was first described by McCullough et al. (19) in 1991, multiple groups have shown this approach to be safe and effective $(4,6,7)$. The general benefits of laparoscopic surgery include less aggressiveness to the abdominal wall with quick recovery, favorable cosmetics, reduced blood loss, low postoperative morbidity and short hospital stay.

The relative contraindications to a laparoscopic surgery include bowel adhesions secondary to multiple previous abdominal surgeries, the need for a concomitant open procedure, and lymphocele anatomy in close proximity to the renal hylum (6).

Since laparoscopy has been employed to treat post transplant lymphocele, a number of complications have been described $(4,8)$. Injury of the urinary tract, particularly of the transplanted ureter, has been described as a major drawback of laparoscopic lymphocele drainage (4), and an incidence rate as high as $20 \%$ has been reported (16). Laparoscopic drainage of small lymphoceles in close proximity to the renal hylum bears an increased risk of iatrogenic ureter or vessel injury.

Recent reviews note different results with regard to complications associated with laparoscopic lymphocelectomy. Hsu et al., in a multicenter review of experienced centers, found a complication rate of 5\% with only a single urinary tract (bladder) injury (8). Cadrobbi et al. (20) found a 7\% incidence of urinary tract injury (renal pelvis, ureter, bladder) with laparoscopic fenestration, but the rate was lower in centers with significant experience.

Proper identification of the renal hylum and the transplanted ureter can be difficult during laparoscopic fenestration. The successful use of intraoperative transcutaneous or laparoscopic ultrasound has also been described (4). The device seems to be a useful adjunct to identify 
structures prior to fenestration and to document complete drainage of the fluid collection. However, the same authors admitted that this tool was not always helpful for identification of the transplant ureter. We believe that meticulous and careful procedure rather than the use of endoscopic ultrasound ensures safety during laparoscopic lymphocele drainage.

Laparoscopic drainage is thought to have a lower incidence of symptomatic recurrences compared with open drainage (10). In the literature the recurrence rate following laparoscopic lymphocele fenestration ranges from $4 \%$ to $6 \%$ $(4,6,8)$. In our series, the recurrence rate after laparoscopy was $4 \%$ ( 1 of 25 ). We believe that it was due to a failure to localize and adequately open a little inferior component of the lymphocele.

Based on our single patient with incomplete drainage of an unappreciated second cavity, we have begun to use laparoscopic transcutaneous ultrasound routinely if there is any question about lymphocele anatomy, in an effort to help prevent further recurrences.

Laparoscopic lymphocele drainage is a safe and effective procedure. Given the benefits of minimally invasive surgery, including reduced postoperative morbidity and shorter hospital stay, we conclude that laparoscopic drainage should be considered first-line therapy for patients with symptomatic post renal transplant lymphoceles.

\section{CONFLICT OF INTEREST}

None declared.

\section{REFERENCES}

1. Burgos FJ, Teruel JL, Mayayo T, Lovaco F, Berenguer A, Orte $\mathrm{L}$, et al.: Diagnosis and management of lymphoceles after renal transplantation. Br J Urol. 1988; 61: 289-93.

2. Howard RJ, Simmons RL, Najarian JS: Prevention of lymphoceles following renal transplantation. Ann Surg. 1976 184: 166-8.

3. Ward K, Klingensmith WC 3rd, Sterioff S, Wagner HN Jr.: The origin of lymphoceles following renal transplantation. Transplantation. 1978; 25: 346-7.
4. Bailey SH, Mone MC, Holman JM, Nelson EW: Laparoscopic treatment of post renal transplant lymphoceles. Surg Endosc. 2003; 17: 1896-9.

5. López García D, Janeiro Pais JM, González Dacal J, Zarraonandía Andraca A, Casas Agudo P, Martínez Breijo S et al.: Lymphocele after renal transplantation: case report and bibliographic review. Arch Esp Urol. 2009; 62: 667-71.

6. Fuller TF, Kang SM, Hirose R, Feng S, Stock PG, Freise CE: Management of lymphoceles after renal transplantation: laparoscopic versus open drainage. J Urol. 2003; 169: 2022-5.

7. Desai MM, Gill IS: Laparoscopic surgery in renal transplant recipients. Urol Clin North Am. 2001; 28: 759-67.

8. Hsu TH, Gill IS, Grune MT, Andersen R, Eckhoff D, Goldfarb DA et al.: Laparoscopic lymphocelectomy: a multi-institutional analysis. J Urol. 2000; 163: 1096-8; discussion 1098-9.

9. Metcalf KS, Peel KR: Lymphocele. Ann R Coll Surg Engl. 1993; 75: 387-92.

10. Braun WE, Banowsky LH, Straffon RA, Nakamoto S, Kiser WS, Popowniak KL et al.: Lymphocytes associated with renal transplantation. Report of 15 cases and review of the literature. Am J Med. 1974; 57: 714-29.

11. Stephanian E, Matas AJ, Gores P, Sutherland DE, Najarian JS: Retransplantation as a risk factor for lymphocele formation. Transplantation. 1992; 53: 676-8.

12. Malovrh M, Kandus A, Buturović-Ponikvar J, Lindic J, Knap B, Fliser $D$ et al.: Frequency and clinical influence of lymphoceles after kidney transplantation. Transplant Proc. 1990; 22: 1423-4.

13. Langer RM, Kahan BD: Incidence, therapy, and consequences of lymphocele after sirolimus-cyclosporine-prednisone immunosuppression in renal transplant recipients. Transplantation. 2002; 74: 804-8.

14. Martínez-Ocaña JC, Lauzurica R, Castellote E, Bonet J, Tenesa M, Jiménez JÁ et al.: Adult polycystic kidney disease: a risk factor for lymphocele formation after renal transplantation? Transplant Proc. 1995; 27: 2246-7.

15. Sansalone CV, Aseni P, Minetti E, Di Benedetto F, Rossetti 0 , Manoochehri $\mathrm{F}$ et al.: Is lymphocele in renal transplantation an avoidable complication? Am J Surg. 2000; 179: 182-5.

16. Kim JK, Jeong YY, Kim YH, Kim YC, Kang HK, Choi HS: Postoperative pelvic lymphocele: treatment with simple percutaneous catheter drainage. Radiology. 1999; 212: 390-4.

17. Manfro RC, Comerlato L, Berdichevski RH, Ribeiro AR, Denicol NT, Berger $M$ et al.: Nephrotoxic acute renal failure in a renal transplant patient with recurrent lymphocele treated with povidone-iodine irrigation. Am J Kidney Dis. 2002; 40: 655-7. 
18. Doehn C, Fornara P, Fricke L, Jocham D: Laparoscopic fenestration of posttransplant lymphoceles. Surg Endosc. 2002; 16: 690-5.

19. McCullough CS, Soper NJ, Clayman RV, So SS, Jendrisak MD, Hanto DW: Laparoscopic drainage of a posttransplant lymphocele. Transplantation. 1991; 51: 725-7.
20. Cadrobbi R, Zaninotto G, Rigotti P, Baldan N, Sarzo G, Ancona $\mathrm{E}$ : Laparoscopic treatment of lymphocele after kidney transplantation. Surg Endosc. 1999;13: 985-90.

\author{
Correspondence address: \\ Dr. Cristiano Augusto Calderaro Cotrim \\ Hospital das Clínicas UNICAMP \\ R. Vital Brazil, 250 - 20andar, A2, sala108 \\ Cidade Universitária Zeferino Vaz \\ Distrito de Barão de Geraldo, \\ Campinas, SP, 13083-590, Brazil \\ E-mail: drcotrim@yahoo.com.br
}

\section{EDITORIAL COMIMENT}

The authors present their experience on the treatment of 25 patients submitted to a kidney transplant with symptomatic lymphoceles. It has been observed a clear reduction of the incidence of lymphoceles in the last decade. There is not an isolate etiology for its occurrence. However, a careful dissection of the iliac vessels with careful taking to ligate the lymphatic vessels is certainly an important step on its prevention.

Before performing the laparoscopic drainage, some steps should be reinforced. A computer tomography is fundamental in planning the surgery, the collection located lateral to the graft or deep in the iliac fossa may represent a major difficult when performing the procedure. The authors have observed just one case of ureteral injury, probably in a patient with a lymphocele deep in the pelvis.

Another important aspect, mentioned by the authors, is to perform a percutaneous aspiration one day before the procedure, to exclude an infected lymphocele. These patients may have an infected collection without any clinical signs.

The laparoscopic access is safe and effective for the treatment of symptomatic lynphocele as shown by the authors.
Dr. William Nahas

School of Medicine USP SP, Brazil

E-mail:wnahas@uol.com.br 